\title{
Zur Lage der Soziologie
}

\section{Zur Selektivität von Herausgebern: Eine Input-output-Analyse der „Zeitschrift für Soziologie“"}

\author{
Heinz Sahner \\ Christian-Albrechts-Univeristät, Institut für Soziologie \\ Olshausenstraße 40-60, D-2300 Kiel 1
}

$\mathrm{Z}$ u s a $\mathrm{m} \mathrm{m}$ e $\mathrm{n}$ f a s $\mathrm{s}$ u $\mathrm{n}$ g: In der Literatur wird häufig behauptet, daß Herausgeber bei der Auswahl von Artikeln gegen das Postulat des Universalismus (Merton) verstoßen. Diese Behauptungen basieren meist auf einer Analyse lediglich der veröffentlichten Arbeiten. In diesem Beitrag werden alle der Zeitschrift für Soziologie zur Veröffentlichung eingereichten Arbeiten analysiert und ihre Veröffentlichungschance nach verschiedenen Merkmalen untersucht. Folgende Ergebnisse seien hervorgehoben: Die Herausgeberpolitik trägt nicht, wie so häufig behauptet wird, zur Artefaktbildung bei, insofern signifikante Befunde und Bestätigungen von Hypothesen keine überdurchschnittliche Diffusionschance aufwcisen. Methodische und makrosoziologische Fragestellungen sowie eine modelltheoretische bzw. metatheoretische Fundierung, die sich an den Naturwissenschaften orientiert, werden favorisiert. Schließlich kann man partikularistische Tendenzen hinsichtlich der Merkmale Hochschulort, Status und Geschlecht vermuten.

\section{Problem}

Forschung kann als mehrstufiger sozialer Prozeß verstanden werden, auf den zahlreiche Faktoren einwirken, mit den entsprechenden Konsequenzen für das innerhalb einer Disziplin angebotene Wissen. Theorieformulierung, Datensammlung, Datenanalyse, Präsentation der Ergebnisse, Rezeption, Diffusion, Verwertung kennzeichnen nur einige wichtige Stufen in diesem Prozeß. Eine besondere Rolle kommt den Herausgebern wissenschaftlicher Zeitschriften zu, und zwar in zweifacher Hinsicht. Einmal berühren sie die Karrierechancen junger Wissenschaftler und zum anderen - und dieser Aspekt soll uns hier vor allem interessieren - wirken sie als gate-kecper in der Wissensproduktion. Das heißt, sie wirken entscheidend am Selbstverständnis der Profession mit. "In sociology today, as in other sciences, the professional journals serve as major channels for the dissemination of ideas and information. The papers presented in the principal periodicals reach a wide audience among sociologists, are acknowledged as the best work, and stand as models for all who aspire to success. The viewpoints, conceptual schemes, interests, and methodologies reflected in these articles are therefore significant influences on both the current character and the future development of our discipline" (Brown und Gilmartin 1969: 283).

Es ist deshalb nicht verwunderlich, daß die Veröffentlichungen wissenschaftlicher Zeitschriften, vor allem aber auch die Herausgeber selbst,
Gegenstand der Analyse von Wissenssoziologen waren. Soweit ich sehe, ist jedoch noch keine Analyse einer wissenschaftlichen Zeitschrift durchgeführt worden, bei der auch die eingereichten Arbeiten einer Inhaltsanalyse unterzogen werden konnten. Dies ist umso erstaunlicher, als den Herausgebern Selektivität unterschiedlichster Art vorgeworfen wurde. Selektive Praktiken können aber nur mittels einer Input-output-Analyse nachgewiesen und nicht etwa lediglich an den Veröffentlichungen selbst abgelesen werden.

Häufig wird ein Ideologieverdacht geäußert und zwar dergestalt, daß theoretische Modelle oder „Ansätze" unterschiedliche Zugangschancen zu dem etablierten Publikationssystem haben (Friedrichs 1970). Ähnlich Matthes (1978: 10): „Als Handlungs- und auch Marktsystem betrachtet, bietet die gegenwärtige Praxis empirischer Sozialforschung bestimmten Theorieansätzen bessere Chancen des Einbringens und der Verwirklichung ihrer Forschungsideen und Forschungsinteressen als anderen, - ein Umstand, der, verwandelt in ein Argument, auf die themenbezogene Diskussion zum Theorievergleich hinderlich zurückwirkt: als ob bestimmte Theorieansätze überhaupt besser für die Forschung geeignet seien als andere. Mit anderen Worten: die themenbezogene Diskussion zum Theorienvergleich wäre einfacher und ertragreicher zu führen, wenn die zeitgenössische Praxis der empirischen Sozialforschung weniger monopolistisch organisiert wäre." Zahlrei- 
che Untersuchungen über "editorial boards" legen den Verdacht nahe, daß gegen den institutionellen Imperativ des Universalismus (Merton 1972) verstoßen wird (vgl. z.B. Beyer 1978). Herausgeber tendieren dazu, Kandidaten für die Herausgeberschaft zu nominieren, die die gleichen Ausbildungsinstitutionen durchlaufen haben wie sie selbst (Yoels 1974; Pfeffer u.a. 1977). Partikularistische Kriterien werden auch bei der Auswahl von Manuskripten vermutet. "As a result of academic training, editiorial readers respond to certain aspects of methodology, theoretical orientation, and mode of expression in the writings of those who have received similar training" (Crane 1967: 200).

Es lassen sich aber auch andere Stimmen vernehmen, die behaupten, "universalism tends to operate in the sense that the institutional affiliation or the standing of authors in the field does not significantly affect editorial and referee decisions" (Zuckerman 1978: 69); und für die Kölner Zeitschrift und für die Soziale Welt behauptet Lüschen (1979: 171), ,daß sie zwar einem bestimmten Institut verbunden sind, sich aber keinesfalls auf eine personelle und sachliche Begünstigung des eigenen Instituts ausgerichtet haben."

Am regelmäßigsten wird eine Selektivität in Verbindung mit Signifikanztests behauptet, nämlich dergestalt, daß signifikante Ergebnisse eine gröBere Diffusionschance hätten als nicht-signifikannte Ergebnisse (vgl. stellvertretend Sterling 1959; Greenwald 1975), was die Produktion von Artefakten in sich birgt (Sahner 1981: 172). Die Analyse von Periodika zeigt auch, daß tatsächlich die signifikanten Ergebnisse überwiegen, jedoch kann man daraus allein nicht auf selektive Publikationschancen schließen (Sahner 1979: 271). Allerdings wurde ein derartiger Verdacht auch durch Herausgeber selbst genährt. Beispielhaft sind hier die Ausführungen von Melton, der in einem ,Editorial" ausfuhrte, daß "in editing the Journal there has been a strong reluctance to accept and publish results to the principle concern of the research when those results were significant at the .05 level, whether by oneor two-tailed test. This has not implied a slavish worship of the .01 level as some critics may have implied. Rather, it reflects a belief that it is the responsibility of the investigator in a science to reveal his effect in such a way that no reason- able man would be in a position to descredit the results by saying that they were the product of the way the ball bounces" (Melton 1962: 553f., zitiert nach Bakan 1966: 427). Dies kann ja nichts anderes heißen, als daß nicht-sifnifikante Ergebnisse kaum eine Chance für eine Aufnahme in das Journal hatten. Diese Haltung kann man sicher nicht verallgemeinern. So wollen Bredenkamp und Feger (1970) diese Tendenz bekämpfen, unterstellen aber offenbar, daß eine derartige Haltung verbreitet ist.

Ob nun selektive Diffusionschancen - die gegen universalistische Prinzipien verstoßen - postuliert werden oder nicht, lediglich aufgrund der Analyse veröffentlichter Forschung lassen sich derartige Aussagen nicht begründen, sondern nur mit Hilfe einer Input-output-Analyse. Und selbst hier sind die Aussagen noch mit Fragezeichen zu versehen, solange man nicht eine Entscheidung bezüglich der Qualität der untersuchten Arbeiten fällen kann oder will.

\section{Die Daten der Untersuchung}

Durch das Entgegenkommen der Herausgeber der Zeitschrift für Soziologie und die hier gepflegten editorischen Standards, die eine genaue Dokumentation der Eingänge und Entscheidungen erfordern, ist die Grundlage für eine derartige Analyse gegeben ${ }^{1}$. Der Untersuchungszeitraum erstreckt sich vom Erscheinen der Zeitschrift im Jahr 1972 bis 1980 einschließlich ${ }^{2}$. Der zeitliche Schnittpunkt für die zu analysierenden eingereichten Manuskripte wurde in Rücksprache mit dem Redakteur festgelegt. Analyseeinheit ist das eingereichte Manuskript bzw.

1 Für diese Möglichkeit danke ich den Herausgebern der Zeitschrift für Soziologie, vor allen Dingen aber Herrn Dr. Klima für seine großzügige Unterstützung.

2 Dieser Aufsatz enthält Material, das unter einer umfassenderen Fragestellung in einer anderen Arbeit des Verfassers erarbeitet worden ist: "Theorie und Forschung. Zur paradigmatischen Struktur der westdeutschen Soziologie und zu ihrem Einfluß auf die Forschung", Kiel 1981 (Habilitationsschrift). Hier ist auch der vollständige Code für die Zeitschriftenanalyse dokumentiert und die Kriterien seiner Erstellung. Neben der Zeitschrift für Soziologie wurden auch die Kölner Zeitschrift und die Soziale Welt jeweils seit Erscheinen analysiert (2114 Aufsätze insgesamt). Weiter enthält diese Arbeit die Analyse der Dissertationen der Schüler ausgewählter Doktorväter. 
der veröffentlichte Aufsatz. Editiorials, Hinweise und Berichte wurden nicht berücksichtigt. Nach dieser Abgrenzung wurden 390 der Zeitschrift eingereichte, aber nicht veröffentlichte und 254 veröffentlichte Manuskripte, insgesamt also 644 Aufsätze analysiert. Danach ergibt sich eine Veröffentlichungsrate von fast vierzig Prozent $(39,44 \%)$, eine im Vergleich zu den Veröffentlichungsraten der angesehensten amerikanischen Fachzeitschriften hohe Chance (vgl. z.B. Simon 1980: 10).

Zuerst sollen nun die Diffusionschancen ${ }^{3}$ für verschiedene Merkmale der Manuskripte und dann für einige ausgewählte Merkmale der Autoren ermittelt werden.

\section{Selektivität gegenuiber Inhalten und Strategien}

\subsection{Die Hierarchie der Forschungsfelder}

Die Forschungsfelder spiegeln das Selbstverständnis der Profession wider. Das, was zur Publikation ausgewählt wird, wirkt selbst aber wiederum beispielhaft und beeinflußt die Entwicklung. Die Herausgeber besitzen hier ein Steuerungspotential, dessen Wirkung freilich schwierig abzuschätzen ist. Sowohl unter den eingereichten als auch unter den veröffentlichten Arbeiten dominieren die theoretischen, methodischen, wissenschaftstheoretischen und wissenschaftssoziologischen ${ }^{4}$ Arbeiten; es dominiert also die Grundlegung der Soziologie. Angewandte und auf konkrete Probleme gerichtete Soziologie folgt erst auf ,,den Plätzen" (Tab. 1). Die Selbstproblematisierung wird besonders an der Inflation wissenschaftstheoretischer Literatur deutlich, die vor allem

3 Unter Diffusionschance wird das Verhältnis der angenommenen zu den eingereichten Arbeiten verstanden.

4 Von den unveröffentlichten Aufsätzen zur Wissenschaftssoziologie entstammt etwa jeder dritte Aufsatz der Kategorie "Soziologie der Soziologie", von den veröffentlichten ist es etwa jeder fünfte. Das Kategorienschema für die "Inhalte" der Aufsätze wurde in Anlehnung an Klima 1979 entwickelt. Bei der Analyse wurde eine Doppelverkodung nach erstem und zweitem Schwerpunkt vorgenommen. Die Analyse in diesem Aufsatz beruht auf der Verkodung nach dem ersten Schwerpunkt. Der vollständige Code und die dazugehörigen Besetzungszahlen befinden sich im Anhang. die Krisenperiode 1965 bis 1975 kennzeichnete (Sahner 1981: $143 \mathrm{ff}$ ) und von der die Zeitschrift für Soziologie nicht verschont worden ist. Diese bevorzugte Beschäftigung mit sich selbst und mit den Grundlagen wird nicht zu Unrecht als ein Zeichen von Schwäche gedeutet (König 1969: 1278; Schelsky 1950: 4). Allerdings läßt sich in den letzten Jahren ein rapider Rückgang der Selbstproblematisierung in Form von wissenschaftstheoretischer Literatur beobachten. Die paradigmatische Ausrichtung der westdeutschen Soziologie auf eine einmal mehr verstehend/geisteswissenschaftlich und zum anderen erklärend/ naturwissenschaftlich operierende Soziologie läßt die Renaissance eines alten Methodenstreites befürchten (vgl. hierzu Sahner 1981: 163).

Gegenüber der „reinen“ Soziologie fallen so zentrale Sachgebiete wie „Makrosoziologie“, „Sozialer Wandel " etc. zurück. Erstaunlich bleibt das geringe Angebot an Manuskripten zur „Politischen Ökonomie", obwohl doch gerade hier in der Analyseperiode die Nachfrage nach gesellschaftlichen „Definitionen der Situation“ und nach allgemeinen „Hintergrundideologien" (Neidhardt 1976: 427) anstieg und mit einer rapiden Vermehrung von Lehrveranstaltungen zur ,Politischen Ökonomie" einherging (Klima 1979: 243). Offenbar war fuir die Vertreter bestimmter Strömungen die Zeitschrift für Soziologie nicht das relevante Publikationsorgan, wie ja von entsprechender Seite auch schon relativ früh Unzufriedenheit mit der Publikationspolitik der Zeitschrift geübt worden ist (Hauck 1972: 377).

Zusammenfassend kann man festhalten: (1) Es läßt sich eine Konzentration auf die Grundlegung beobachten, nämlich auf allgemeine soziologische Theorie, Konzeptualisierung, Interpretation der Klassiker und auf die Methoden. (2) Deutlich zeichnet sich eine Selbstproblematisierung in Form der Wissenschaftssoziologie ab, die zu einem beträchtlichen Teil eine Soziologie der Soziologie ist und die sich vor allem in den zahlreichen wissenschaftstheoretischen Beiträgen manifestiert. Erst weiter unten in der Rangordnung folgt eine an Inhalten orientierte Soziologie.

Doch wird aus der Hierarchie der Diffusionschan cen ein Bemuihen der Herausgeber deutlich, dieser Tendenz entgegen zu wirken (Tab. 1). Die ersten drei Plätze nehmen inhaltliche Fragestellun- 
TABELLE 2: Diffusionschancen und Art der Forschung*

\begin{tabular}{|c|c|c|c|c|c|c|c|}
\hline & \multicolumn{6}{|c|}{ Zeitschrift für Soziologie 1972 bis 1980} & \multirow{2}{*}{$\begin{array}{l}\text { Diff.-Chance } \\
\%\end{array}$} \\
\hline & $\begin{array}{l}\text { einger } \\
\mathrm{N}\end{array}$ & $\%$ & $\begin{array}{l}\text { veröff } \\
\mathrm{N}\end{array}$ & $\begin{array}{c}\text { Fentl. } \\
\%\end{array}$ & $\begin{array}{l}\text { nicht } \\
\mathbf{N}\end{array}$ & $\begin{array}{l}\text { ver. } \\
\%\end{array}$ & \\
\hline theoretisch & 403 & 63,0 & 138 & 54,3 & 265 & 68,7 & 34,2 \\
\hline methodisch & 94 & 14,7 & 56 & 22,0 & 38 & 9,8 & 59,6 \\
\hline $\begin{array}{l}\text { Substanzforsch. } \\
\text { insgesamt }\end{array}$ & 143 & 22,3 & 60 & 23,6 & 83 & 21,5 & 42,0 \\
\hline $\begin{array}{l}\text { Substanzforsch. } \\
\text { o. Signif.-Test }\end{array}$ & 86 & 13,4 & 26 & 10,2 & 60 & 15,5 & 30,2 \\
\hline $\begin{array}{l}\text { Substanzforsch. } \\
\text { m. Signif.-Test }\end{array}$ & 57 & 8,9 & 34 & 13,4 & 23 & 6,0 & 59,6 \\
\hline & 640 & & 254 & & 386 & & 39.7 \\
\hline
\end{tabular}

* In den Tabellen 2 bis 8 gingen vier Aufsätze weniger in die Analyse ein, für die die relevanten Informationen (nichtveröffentlichte Manuskripte) nicht mehr vorlagen.

TABELLE 3: Datenanalyse und Diffusionschancen

\begin{tabular}{|c|c|c|c|c|c|c|c|}
\hline & \multicolumn{7}{|c|}{ Zeitschrift für Soziologie 1972 bis 1980} \\
\hline & \multicolumn{2}{|c|}{ einger. $\%$} & \multicolumn{2}{|c|}{ veröffentl. } & \multicolumn{2}{|c|}{$\begin{array}{l}\text { nicht ver. } \\
\mathbf{N} \%\end{array}$} & $\begin{array}{l}\text { Diff.-Chance } \\
\%\end{array}$ \\
\hline Allg. Interpret. & 22 & 12,1 & 6 & 7,1 & 16 & 16,5 & 27,3 \\
\hline Randverteilung & 12 & 6,6 & 2 & 2,4 & 10 & 10,3 & 16,7 \\
\hline 2-Var.-Analyse & 69 & 37,9 & 33 & 38,8 & 36 & 37,1 & 47,8 \\
\hline 3-Var.-Analyse & 4 & 2,2 & 3 & 3,5 & 1 & 1,0 & 75,0 \\
\hline Faktorenanalyse & 11 & 6,0 & 7 & 8,2 & 4 & 4,1 & 63,6 \\
\hline Varianzanalyse & 13 & 7,1 & 7 & 8,2 & 6 & 6,2 & 53,8 \\
\hline $\begin{array}{l}\text { Regressions- } \\
\text { analyse }\end{array}$ & 18 & 9,9 & 9 & 10,6 & 9 & 9,3 & 50,0 \\
\hline Pfadanalyse & 14 & 7,7 & 8 & 9,4 & 6 & 6,2 & 57,1 \\
\hline $\begin{array}{l}\text { And. Multiv. } \\
\text { Verf. }\end{array}$ & 19 & 10,4 & 10 & 11,8 & 9 & 9,3 & 52,6 \\
\hline & 182 & & 85 & & 97 & & 46,7 \\
\hline
\end{tabular}

reichen eine gleich hohe Annahmewahrscheinlichkeit wie Methodenartikel.

Letztere Vermutung wird gestützt durch die Daten der Tab. 3. Während Aufsätze mit allgemeinen Interpretationen und Diskussionen von Randverteilungen eine weit unter dem Durchschnitt liegende Diffusionschance haben und die 2-Variablenanalyse den Durchschnittswert gerade übersteigt, liegen alle Beiträge mit multivariaten Analyseverfahren weit bzw. deutlich über dem Durchschnitt.

Gewisse methodische Standards mögen bei den Entscheidungen über die Aufnahme von Manuskripten eine Rolle gespielt haben. Daß die Substanzforschung jedoch eine relativ bescheidene Rolle in der veröffentlichten Forschung spielt, geht wohl weniger auf die Selektionsfunktion der Herausgeber zurück, sondern dürfte vorwie- gend die Angebotssituation widerspiegeln. Selbst wenn die Herausgeber der Zeitschrift für Soziologie alle eingereichten empirischen Arbeiten publiziert hätten, wäre der Anteil der Substanzforschung nur auf $56,3 \%$ angestiegen und hätte damit noch deutlich unter dem gelegen, den Brown und Gilmartin für die AJS und die ASR für die Jahre 1965 und 1966 errechnet haben $(69,8 \%)^{6}$. Bei den Zahlen für die Zeitschrift für Soziologie muß man freilich bedenken, daß die meisten Jahre ihres Erscheinens in eine Periode fielen, in der sich die Soziologie in der berühmten „Krise" befand (Gouldner 1974; Eisermann 1976; König 1979: 345 ff; Krysmanski/Marwedel 1975; Luckmann 1974 usw.) und die durch eine drastische Reduktion der empirischen Forschung - zumindest der veröffentlichten - gekennzeichnet war (vgl. hierzu Sahner 1981: 142).

Unterstellt man den Herausgebern nicht nur die Intention, lediglich nach Qualitätsgesichtspunkten zu entscheiden, sondern auch ein entsprechendes Verhalten ${ }^{7}$, dann ist nach den Diffusionschancen Kompetenz und Qualität am ehesten bei methodischen Arbeiten zu vermuten ${ }^{8}$, ein weiterer Indikator für die stürmische Entwicklung gerade der Methoden, die aber offenbar nicht für inhaltlich relevante Substanzforschung fruchtbar gemacht wird. Möglicherweise hat sich die Soziologie schon so weit ausdifferenziert, daß methodische und theoretische Kompetenz nicht mehr so ohne weiteres in empirische Forschung umgesetzt werden kann.

\subsection{Modelltheoretische und meta-theoretische Fundierung}

„Beide, Herausgeber und Autoren, reproduzieren in der Zeitschrift den Spiegel des eigenen Fa-

6 Auch Gaston (1979), Wiley (1979) und Zelditch (1979), die die drei großen amerikanischen Zeitschriften "American Journal of Sociology", "American Sociological Review" und "Social Forces" der Jahre 1975 bis 1977 besprechen, betonen die Dominanz empirischer Beiträge in diesen Zeitschriften.

7 Was sicher nicht ganz realistisch ist, denn man wird z.B. bestrebt sein, ein vorteilhaft erscheinendes Verhältnis zwischen theoretischen, methodischen und empirischen Arbeiten zu erreichen.

$8 \mathrm{Vgl}$. hierzu auch die interessanten Ausfihrungen bei Hartmann 1979: 3. 
ches" (Hartmann 1979: 4). Diese Meinung ist unter Herausgebern verbreitet. Dieser Tenor herrscht auch bei Gaston (1979) und den meisten anderen Autoren des Sonderheftes der Zeitschrift "Contemporary Sociology" vor, in dem drei Jahrgänge der AJS, ASR und Social Forces impressionistisch analysiert werden. Die Aussage von Hartmann ist aber zumindest diskussionsbedürftig. Denn auch wenn man mit einigem Recht sagen kann, daß die drei repräsentativen bundesrepublikanischen Zeitschriften die Forschung relativ breit widerspiegeln, so dürfte sich das wenn überhaupt - höchstens auf die Inhalte beziehen. Ist es darüber hinaus aber nicht vielmehr so, daß je nach Fundierung der Soziologie spezifische Publikationsmedien sich ausgebildet haben? Erst die Fachorgane insgesamt könnten danach das Selbstverständnis der Profession widerspiegeln. Diskussionsbedürftig bleibt diese Behauptung auch deshalb, weil es an Input-output-Analysen mangelt; daran ändern auch die mittlerweile zahlreichen Befragungen von Herausgebern nichts (vgl. z.B. Lindsey 1978).

Wie leicht es zu Fehleinschätzungen selbst hinsichtlich der veröffentlichten Forschung kommen kann, zeigt die These von Lepsius (1979: 51), wonach der Strukturfunktionalismus die stärkste theoretische Orientierung blieb. Dieser wurde aber nach einer Analyse der Veröffentlichungen der Kölner Zeitschrift, der Sozialen Welt und der Zeitschrift für Soziologie ab Mitte der sechziger Jahre zuerst von der Kritischen Theorie und dann zu Beginn der siebziger Jahre auch noch vom Historischen Materialismus/Marxismus übertroffen. Heute sind allerdings diese beiden theoretischen Orientierungen zugunsten des interpretativen Paradigmas weit hinter den Funktionalismus zurückgefallen (Sahner 1981: Abb. 4.2.1.: 1)

Da das, was den Herausgebern zur Publikation angeboten wird, wiederum kaum ein getreues Spiegelbild der Forschung sein dürfte, kann man die These von Hartmann so ohne weiteres nicht auf ihren Wahrheitsgehalt hin überprüfen. Dagegen kann eine Antwort auf die Frage gegeben werden, ob die publizierte Forschung das Manuskriptangebot widerspiegelt. Von besonderem Interesse ist hier selbstverständlich die modelltheoretische und meta-theoretische Fundierung der Beiträge ${ }^{9}$.

9 Eine Einordnung wird nach Selbstdefinition des Autors bzw. nach den die einzelnen Richtungen konstituierenden Merkmalen vorgenommen, soweit sie
Richtig ist sicher, daß das, was in die Zeitschriften Eingang findet, durch das Angebot begrenzt wird. Nur aus dem Autorenangebot kann ausgewählt werden. Der Einfluß der Herausgeber (und Gutachter) läßt sich aber deutlich an den Diffusionschancen einzelner theoretischer Modelle und Meta-Theorien ablesen. Anhand der in Tab. 4 dargestellten Ergebnisse kann man wohl kaum noch davon sprechen, daß Herausgeber und Autoren in der Zeitschrift den Spiegel des eigenen Faches reproduzieren.

Für den Untersuchungszeitraum, der deutlich durch die Dominanz der Kritischen Theorie und des Historischen Materalismus geprägt war, wurden auch der Zeitschrift für Soziologie Manuskripte mit entsprechender Fundierung angeboren. Von den Artikeln, die überhaupt eine modelltheoretische und meta-theoretische Fundierung erkennen ließen, nahmen diejenigen, die sich an die Kritische Theorie und an den Historischen Materialismus anlehnten, die Spitzenstellung ein (Tab. 4a). Funktionalismus und Verhaltenstheoretische Soziologie rangierten hinter Modellen, die man zusammenfassend als dem interpretativen Paradigma zugehörig bezeichnen kann (Verstehen, Handlungstheorie, Phänomenologie, Symbolischer Interaktionismus). Unter den veröffentlichten Manuskripten wird diese Hierarchie aber nahezu umgekehrt. Von den 64 Aufsätzen, deren Autoren sich der Kritischen Theorie verpflichtet fühlten, wurden lediglich fünf in der Zeitschrift für Soziologie veröffentlicht. Nicht viel anders sieht es beim Historischen Materialismus aus. Dagegen nehmen nun unter den veröffentlichten Aufsätzen die Modelle Logischer Empirismus, Systemtheorie, Funktionalismus und Kritischer Rationalismus Spitzenstellungen ) ein.

\section{Nach der Hierarchie der Diffusionschancen} (Tab. 4c) werden die Präferenzen der Herausgeber am deutlichsten. Danach haben die Autoren, die sich mehr einem naturwissenschaftlichen $\mathrm{Pa}$ radigma verpflichtet fühlen, die größten Chancen, daß ihre Manuskripte veröffentlicht werden, gefolgt von denen, die mehr ein interpretatives Paradigma pflegen. Das Schlußlicht bilden Aufsätze, deren Autoren eher eine marxistische

sich in den analysierten Texten niedergeschlagen haben. Zur Entwicklung des Codes vgl. Sahner 1981, bes. Kap. 2.1 . 
TABELLE 4: Hierarchie der verwendeten theoretischen Modelle und Meta-Theorien in der Zeitschrift für Soziologie 1972-1980 (Mehrfachverkodung möglich)

a) Eingereichte Manuskripte

\begin{tabular}{lrrl} 
& N & \multicolumn{1}{c}{$\%$} & \\
\hline 1. Kritische Theorie & 64 & 13,4 & 1. Logischer Empir. \\
2. Histor. Material. & 53 & 11,1 & 2. Systemth., Kyb. \\
3. Verstehen & 49 & 10,3 & 3. Funktionalismus \\
4. Logischer Empirism. & 46 & 9,7 & 4. Krit. Rational. \\
5. Kritischer Ration. & 37 & 7,8 & 5. Kritik und Disk.* \\
6. Systemtheorie, Kyb. & 37 & 7,8 & 6. Verstehen \\
7. Kritik und Disk.* & 32 & 6,7 & 7. Handlungstheorie \\
8. Handlungstheorie & 31 & 6,5 & 8. Phänomenologie \\
9. Phänomenologie & 28 & 5,9 & 9. Verhaltensth. Soz. \\
10. Symbol. Interakt. & 24 & 5,0 & 10. Histor. Mater. \\
11. Funktionalismus & 23 & 4,8 & 11. Kritische Theorie \\
12. Verhaltenstheorie & 23 & 4,8 & 12. Symb. Interakt. \\
13. Psychoanalyse & 14 & 2,9 & 13. Ethnomethodol. \\
14. Ethnomethodologie & 8 & 1,7 & 14. Psychoanalyse \\
15. Konflikttheorie & 7 & 1,5 & 15. Konflikttheorie \\
16. Beziehungslehre & 0 & 0,0 & \\
\hline
\end{tabular}

c) Diffusionschance der veröffentlichten Arbeiten

* Hier wurden Artikel subsumiert, die sich kritisch mit den hier aufgeführten theoretischen Modellen und Meta-Theorien auseinandersetzten.

Grundlegung erkennen lassen. Die Förderung eines naturwissenschaftlichen Soziologieverständnisses ist augenfallig. Die Autonomie der Herausgeber ist zwar durch das Angebot von Manuskripten beschränkt, doch innerhalb dieses Angebotes gibt es ausreichenden Spielraum zur Förderung eines bestimmten Wissenschaftsverständnisses, der von den Herausgebern auch wahrgenommen wird.

Die geringe Vertretung des marxistischen Ansatzes blieb auch den Herausgebern nicht verborgen: ,Wir sind uns bewußt, daß wir bisher kaum qualifizierte Arbeiten marxistisch orientierter Autoren publizieren konnten. $\mathrm{Da}$ uns diese $\mathrm{Au}$ toren relativ selten Beiträge anbicten, dürfte teilweise daran liegen, daß das Publikum, an das sie sich in erster Linie wenden, eher über spezialisierte Publikationsorgane zu erreichen ist als über eine breit orientierte soziologische Fachzeitschrift. Wenn wir ,linke" Manuskripte zur Veröffentlichung angeboten erhalten, dann handelt es sich leider nur allzu oft um offensichtliche Gelegenheitsarbeiten oder jedenfalls um Beiträge von eindeutig minderer Qualität, mit denen die Verfasser vermutlich bei anderen Zeitschriften auch kein Glück hätten“ (Kaufmann et al. 1975: 3f). Wohlgemerkt, es wird nicht behauptet, daß kaum marxistisch beeinflußte Manuskripte angeboten werden, sondern daß kaum qualifizierte Manuskripte angeboten werden. Damit wären die selektiven Diffusionschancen zumindest teilweise durch Qualifikationsdefizite zu erklären, ein Argument, das möglicherweise auch für die Vertreter eines interpretativen Paradigmas zuträfe, obwohl hierzu von den Herausgebern keine Ausführungen gemacht werden. Dieses Argument zu entkräften oder zu bestätigen ist so einfach nicht. $\mathrm{Zu}$ überlegen ist jedoch, wieweit man sich wiederum auf Kriterien eines bestimmten Wissenschaftsverständnisses stützen muß, um ein derartiges Urteil fällen zu können.

Ein weiterer Versuch, daß Diffusionsgefälle zu erklären, sei angefügt. Die Zeitschrift für Soziologie hat als einzige der drei großen Fachorgane einen klassischen Gutachterprozeß, wie er z.B. in angelsächsischen Zeitschriften gang und gäbe ist: Mehrere Herausgeber, einen Herausgeberbeirat und darüber hinaus separat rekrutierte Gutachter. Zudem wird der Entscheidungsprozeß dokumentiert. Es dürfte außer Zweifel stehen, $\mathrm{da} ß$ es sich jeweils um angesehene Vertreter des Faches handelt, die die augenblicklich geltenden Standards praktizieren und auch nach außen vertreten. Möglicherweise ist gerade dieses System für Innovationen - und der Untersuchungszeitraum ist ja durch einen Anstieg erst des marxistischen und dann des interpretativen Paradigmas gekennzeichnet - besonders undurchlässig. Ein Autor, der hinsichtlich Problemwahl, theoretischer Fundierung und Analyseverfahren 
von den etablierten Standards abweicht, bleibt in einem derartigen Netz eher hängen als bei einem einzelnen Herausgeber. Vergleicht man nämlich die Hierarchie der vertretenen Fundierungen mit denen der Kölner Zeitschrift und der Sozialen Welt (Sahner 1981: 179), so zeigt sich in diesen Periodika für die Untersuchungsperiode eine Dominanz des interpretativen und des marxistischen Paradigmas (Kritische Theorie, Historischer Materialismus). Selbstverständlich läßt das keine Rückschlüsse auf die spezifische Selektivität in diesen Periodika zu, da der Input unbekannt ist.

\subsection{Favorisierung signifikanter Befunde und Bestätigungstendenz?}

"Editorial policies favor those studies which are successful in rejecting null hypotheses". Bloxham (1976: 135) ist nur ein Zeuge für diese generell vorherrschende Meinung, daß Herausgeber signifikante Befunde favorisieren.

Dieser Vorwurf hat eine lange Tradition, und er ist deshalb von Bedeutung, weil für den Fall, $\mathrm{da} B$ er eine Berechtigung hat, in den Periodika ein nicht näher zu beziffernder Anteil von Kunstprodukten enthalten ist; denn selbst für den Fall, daß in der Grundgesamtheit gar keine Beziehung z.B. zwischen zwei Variablen besteht, dann wird bei dem herkömmlichen Signifikanzniveau von $p=0,05$ trotzdem im Massenexperiment in fünf von hundert Fällen ein signifikantes Ergebnis zu erwarten sein. Wenn diese signifikanten Ergebnisse eine größere Publikationschance besitzen, dann werden Artefakte produziert.

Bezugspunkt für diese Vorwürfe ist ein Beitrag von Sterling (1959), wonach in etwa 97\% der analysierten Artikel signifikante Ergebnisse überwiegen bzw. die Hauptnullhypothese widerlegt worden ist. Andere Studien mit ähnlichen Ergebnissen folgten (vgl. z.B. Galtung 1967: 360f; Wilson et al. 1973). Seither herrscht bei vielen der Eindruck vor, daß statistische Signifikanz eine notwendige Voraussetzung für die Publikation ist.

Umfassendere Untersuchungen der drei dominanten deutschen Periodika ergaben eine weniger krasse Relation zwischen signifikanten und nicht signifikanten Ergebnissen, doch überwiegen auch hier die signifikanten Befunde (Sahner 1979).
Die Analyse aller Jahrgänge der drei Zeitschriften bis einschließlich Jahrgang 1980 zeigt sogar ein noch ausgeglicheneres Verhältnis, was vor allen Dingen auf Veränderungen in den letzten Jahren zurückzuführen ist, wie sich aus einer jahrgangsspezifischen Auswertung ergibt (Sahner 1981: 185ff). Aber auch hier überwiegen nach wie vor die signifikanten Befunde. Und diese Dominanz wurde immer wieder als Beleg für die Favorisierung signifikanter Ergebnisse durch Autoren, aber auch durch die Herausgeber angeführt. Typisch ist eine Formulierung von Becker: "The assumption of equal probability of publication is important precisely because, as Sterling's figures showed, it is systematically violated" (1970: 21). Sterling hat aber nur die veröffentlichten Studien untersucht, und daran kann man nicht ablesen, welche Publikationswahrscheinlichkeiten bestimmte Forschungsbefunde haben. Offenbar ist nach der Meinung von Becker eine gleiche Publikationschance bei einem ausgeglichenen Verhältnis von signifikanten zu nicht-signifikanten Ergebnissen gegeben. Auch Sterling gibt zu Mißverständnissen Anlaß, wenn er schreibt: "Table 31 shows that for psychological journals a policy exists under which the vast majority of published articles satisfy a minimum criterion of significance" (1959: 31). Derartige Relationen lassen aber solche Schlüsse nicht zu, denn sie sind von einer Reihe weiterer Faktoren abhängig. So dürfte die Nullhypothese kaum jemals exakt zutreffen. In der Grundgesamtheit wird es zwischen zwei Parametern Unterschiede geben, wenn auch noch so kleine. Indifferenz zwischen zwei Variablen dürte ebenfalls kaum auftreten. Wenn das der Fall ist, dann wird bei genügend großer Fallzahl immer mit signifikanten Ergebnissen zu rechnen sein. Kurz, derartige Relationen, wie immer sie ausfallen, lassen keine begründeten Rückschlüsse auf die Selektivität der Autoren und der Herausgeber zu.

Die vorliegende Analyse bestätigt für die Zeitschrift für Soziologie die schon früher geäußerte Vermutung (Sahner 1979: 271), daß nämlich die Dominanz signifikanter Befunde nicht durch die Herausgeber verursacht wird. Es ist vielmehr so, daß die Diffusionschance nichtsignifikanter Ergebnisse größer ist als die signifikanter Befunde. Wählt man als Analyseeinheit Artikel, dann haben diejenigen mit überwiegend signifikanten Ergebnissen eine Diffusionschance von $56,1 \%$, wohingegen diejenigen mit überwiegend nicht-signi- 
TABELLE 5: Herausgeber als "gate-keeper" für signifikante Befunde. Erhebungseinheit: Artikel

Alle der Zeitschrift für Soziologie eingereichten Arbeiten

$\mathrm{N}$
Von der Zeitschrift für

Soziologie veröffentlichte

Arbeiten
Diffusionschance

$\% \quad \%$
Artikel mit überwiegend signifikanten Ergebnissen

Artikel mit überwiegend nichtsignifikanten Ergebnissen

Ausgeglichenes Verhältnis
5
N

$\% \quad \mathrm{~N}$

62,2

56,1

$69,5 \quad 23$

$22,0 \quad 11$

$8,5 \quad 3$

29,7

8,1
84,6

60,0

59

$100,0 \quad 37$

100,0

Herausgeber als “gate-keeper” für signifikante Befunde. Erhebungseinheit: Signifikanztest

\begin{tabular}{llllll}
\hline & \multicolumn{2}{l}{$\begin{array}{l}\text { Alle der Zeitschrift für } \\
\text { Soziologie eingereichten } \\
\text { Arbeiten }\end{array}$} & $\begin{array}{l}\text { Von der Zeitschrift für } \\
\text { Soziologie veröffentlichte } \\
\text { Arbeiten }\end{array}$ & Diffusionschance \\
& N & $\%$ & N & $\%$ & $\%$ \\
\hline $\begin{array}{l}\text { Signifikante Ergebnisse } \\
\begin{array}{l}\text { Nicht-signifikante } \\
\text { Ergebnisse }\end{array}\end{array}$ & 1712 & 60,3 & 1107 & 54,8 & 64,7 \\
\hline $\begin{array}{l}\text { Signifikanztests ins- } \\
\text { gesamt }\end{array}$ & 1126 & 39,7 & 913 & 45,2 & 81,1 \\
\hline
\end{tabular}

fikanten Ergebnissen eine Diffusionschance von immerhin $84,6 \%$ haben (Tab. 5). Ähnlich liegt der Fall, wenn man als Erhebungseinheit den einzelnen Signifikanzstest wählt. Hier liegt die Diffusionschance bei $65 \%$ für signifikante und bei $81 \%$ für nicht-signifikante Ergebnisse (Tab. 6) ${ }^{10}$.

Es gibt auch keine Anzeichen dafür, daß die Herausgeber einer Bestätigungstendenz ex-ante formulierter Hypothesen unterliegen. Artikel mit

10 Allerdings muß hier angemerkt werden, daß in die Tabelle 6 ein Artikel, in dem exorbitant viele Tests durchgeführt wurden, nicht einbezogen wurde. Die Anzahl von 2576 Tests (!) in diesem einen Aufsatz übersteigt die, die sich in allen anderen veröffentlichten Aufsätzen befindet, und reicht fast an die Zahl heran, die sich in allen der Zeitschrift für Soziologie eingereichten Manuskripten findet (vgl. Tabelle 6). Von den 2576 Tests waren 859 signifikant und 1717 nicht-signifikant. Der Artikel wurde nicht zur Veröffentlichung angenommen. Berücksichtigt man diese Befunde, dann kommt man für die Ebene der Signifikanztests zu einen grundlegend anderen Ergebnis, wie man leicht mit Hilfe der Ta- überwiegend bestätigten Hypothesen haben eine Diffusionschance von rund $61 \%$, die mit überwiegend widerlegten Hypothesen von rund $71 \%$ (Tab. 7). Wählt man als Analyseeinheit die einzelne ex-ante formulierte Hypothese, so liegen die entsprechenden Anteile bei $54 \%$ bzw. $80 \%$ (Tab. 8).

Freilich muß man sich hüten, die Ergebnisse zu verallgemeinern, aber es besteht Anlaß, von dem Stereotyp Abschied zu nehmen, daß Herausge-

belle 6 errechnen kann, denn dann hätten die signifikanten Ergebnisse die immer wieder behauptete größere Diffusionschance von in diesem Fall 43,1\%. Die nicht-signifikanten Ergebnisse hätten eine kleinere von $32,1 \%$. Aufgrund der extrem hohen Zahlen, die alle anderen Befunde dominieren, wurden die Ergebnisse dieser Untersuchungseinheit nicht mit in die Tabelle 6 aufgenommen. Wer will, der kann darin eine Manipulation erkennen. Ich glaube allerdings, daß die Gründe für die Verfahrensweise stichhaltig sind. Zudem stützt Tabelle 5 nach wie vor die These, daß die Herausgeber signifikante Ergebnisse nicht favorisieren. In diese Tabelle ist die genannte Untersuchung selbstverständlich eingegangen. 
TABELLE 7: Herausgeber als "gate-keeper" für ex ante formulierte Hypothesen. Erhebungseinheit: Artikel

\begin{tabular}{|c|c|c|c|c|c|}
\hline & \multicolumn{2}{|c|}{$\begin{array}{l}\text { Alle der Zeitschrift für } \\
\text { Soziologie eingereichten } \\
\text { Arbeiten }\end{array}$} & \multicolumn{2}{|c|}{$\begin{array}{l}\text { Von der Zeitschrift für } \\
\text { Soziologie veröffentlichte } \\
\text { Arbeiten }\end{array}$} & \multirow{2}{*}{$\begin{array}{c}\text { Diffusionschance } \\
\%\end{array}$} \\
\hline & $\mathbf{N}$ & $\%$ & $\mathbf{N}$ & $\%$ & \\
\hline $\begin{array}{l}\text { Artikel mit überwiegend } \\
\text { bestätigten Hypothesen }\end{array}$ & 36 & 80,0 & 22 & 78,6 & 61,1 \\
\hline $\begin{array}{l}\text { Artikel mit überwiegend } \\
\text { widerlegten Hypothesen }\end{array}$ & 7 & 15,6 & 5 & 17,9 & 71,4 \\
\hline Ausgeglichenes Verhältnis & 2 & 4,4 & 1 & 3,5 & 50,0 \\
\hline Insgesamt & 45 & 100,0 & 28 & 100,0 & \\
\hline
\end{tabular}

TABELLE 8: Herausgeber als "gate-keeper" für ex ante formulierte Hypothesen. Erhebungseinheit: Hypothese

\begin{tabular}{|c|c|c|c|c|c|}
\hline & \multicolumn{2}{|c|}{$\begin{array}{l}\text { Alle der Zeitschrift für } \\
\text { Soziologie eingereichten } \\
\text { Arbeiten }\end{array}$} & \multicolumn{2}{|c|}{$\begin{array}{l}\text { Von der Zeitschrift für } \\
\text { Soziologie veröffentlichte } \\
\text { Arbeiten }\end{array}$} & $\begin{array}{c}\text { Diffusionschance } \\
\%\end{array}$ \\
\hline Bestätigte Hypothesen & 98 & 66,7 & 53 & 57,6 & 54,1 \\
\hline Widerlegte Hypothesen & 49 & 33,3 & 39 & 42,4 & 79,6 \\
\hline Insgesamt & 147 & 100,0 & 92 & 100,0 & \\
\hline
\end{tabular}

ber signifikante Befunde favorisieren oder Studien, in denen die Arbeitshypothesen bestätigt werden, eine größere Annahmechance einräumen. Vielleicht hat dieses Ergebnis positive Rückwirkungen auf die Autoren, denn die Einzelanalyse der Aufsätze stützte die Vermutung (Sahner 1981: 189ff), daß zahlreiche Autoren eine Vorliebe für signifikante Ergebnisse und für ihre Arbeitshypothesen haben.

\section{Selektivität gegenüber Personen}

„Denn wer hat, dem wird gegeben werden, und er wird Uberfluß haben; wer aber nicht hat, dem wird auch noch, was er hat, genommen werden" (Matthäus 13, Vers 12). Auf diesen Vers bezieht sich wohl Merton (1973: 445; zuerst 1968) wenn er vom Matthäus-Effekt spricht, wonach ein Wissenschaftler von Rang für eine Leistung mehr Anerkennung gewinnt als ein wenig angesehener $^{11}$. Die Gültigkeit dieser These würde einmal mehr der wissenschaftlichen Norm des Uni- versalismus widersprechen, wonach die Annahme oder Ablehnung der (Wahrheits-)Ansprüche nicht von personalen oder sozialen Eigenschaften ihrer Protagonisten abhänge und Rasse, Nationalität, Religion, Klassenzugehörigkeit oder persönliche Qualitäten in diesem Zusammenhang irrelevant seien (Merton 1972: 48, zuerst 1942). Tatsächlich gibt es aber zahlreiche Anhaltspunkte für die Wirksamkeit partikularistischer Momente im Wissenschaftsprozeß. Merton wäre sicher der letzte, der dies bestritte. Die Literatur hierzu ist umfangreich (vgl. beispielhaft Merton 1973; Cole/ Cole 1973; Gaston 1978). Im folgenden soll kurz auf die Bedeutung der Merkmale Geschlecht, Status und Hochschulort eingegangen werden, die immer wieder als relevant hervorgehoben werden. Freilich können dabei Drittvariablen eine Rolle spielen, deren Bedeutung man hier nicht kontrollieren kann. So läßt sich das Argument, daß durchschnittlich mit steigendem Status eine qualitativ hochwertigere Forschung zu erwarten ist, kaum überzeugend entkräften (vgl. z.B. Bolte 1978: 13).
11 Die von Merton hier zugrunde gelegte Interpretation des Apostels Matthäus ist aber eine sehr private Deutung. Vgl. hierzu: Neues Testament, 1954: 60, Anmerkung zu Vers 12.

\subsection{Hochschulort}

Die These von Lüschen (1979: 171), daß weder die Soziale Welt noch die Kölner Zeitschrift sich 
TABELLE 9: Hochschulort der Autoren und Diffusionschance

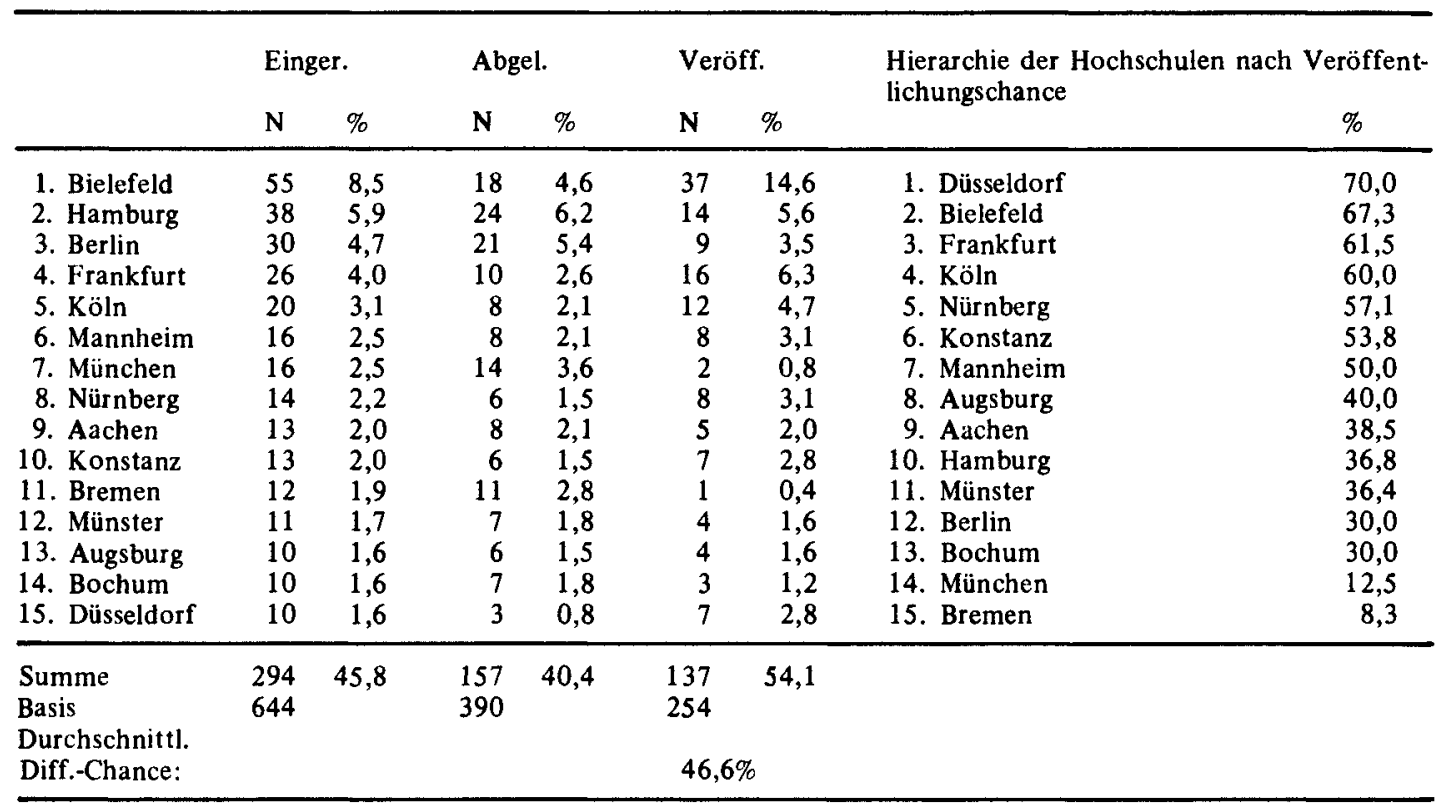

auf eine personelle und sachliche Begünstigung des eigenen Instituts ausgerichtet haben, wird von den Herausgebern auch auf die Zeitschrift für Soziologie ausgedehnt (Flora et al. 1981: 3). Letztere bezog Lüschen wohl deshalb nicht mit ein, weil sie durch die besondere Konstruktion der Herausgeberschaft (vgl. Baier et al. 1972: 3) nicht so deutlich einem bestimmten Institut zuzuordnen ist und daher weniger leicht in einen entsprechenden Verdacht geraten konnte.

Betrachtet man die Autoren nach ihrem Hochschulort $^{12}$, dann nimmt Bielefeld, sowohl was die eingereichten Arbeiten als auch was die veröffentlichten Artikel betrifft, eine Spitzenstellung ein (Tab. 9). Von keinem anderen Hochschulort wurden mehr Manuskripte eingereicht bzw. veröffentlicht, obwohl Bielefeld hinsichtlich der Zahl der Lehrstühle für Soziologie hinter Berlin und Frankfurt erst an dritter Stelle steht (einschließlich TU, PH) und auch kaum einen umfangreicheren Mittelbau haben dürfte als diese. Dieses Ergebnis ist aber so überraschend nicht, weil die potentiellen Autoren vor Ort erst einmal ,ihrer" Zeitschrift ein Manuskript zur Veröffentlichung anbieten dürften.

$1287 \%$ aller Autoren stammen aus dem Hochschulbereich! Dies sieht bei der Kölner Zeitschrift und der Sozialen Welt nicht viel anders aus.
Eine personelle Begünstigung könnte man aber in der Diffusionschance Bielefelder Autoren sehen. Bei der durchschnittlichen Diffusionschance der berücksichtigten fünfzehn ${ }^{13}$ Hochschulen liegt Bielefeld mit $67,3 \%$ weit über dem Durchschnitt und wird nur noch von Düsseldorf übertroffen. Der mögliche Einwand, daß man die aus der Bielefelder Fakultät rekrutierten Herausgeber aus dieser Zählung aussparen müßte (so wurden z.B. alle acht von Luhmann eingereichten Arbeiten auch angenommen), zählt nicht, denn erstens dokumentiert sich so ja ein spezielles Lokalkolorit und zweitens werden nach den selbst gesetzten Regeln Beiträge von Herausgebern ,selbstverständlich den gleichen Veröffentlichungsbedingungen und Prüfverfahren unterworfen wie diejenigen anderer Autoren" (Baier et al. 1974: 1). Will man für die 55 Bielefelder Beiträge nicht eine spezielle Qualität reklamieren, dann bleibt der Verdacht einer personellen Begünstigung ,,des eigenen Instituts".

\subsection{Akademischer Status}

Entsprechend der Mertonschen These vom Matthäus-Effekt müßten die Autoren mit einem

13 Es wurden nur die Hochschulen berücksichtigt, von denen aus mindestens 10 Manuskripte eingereicht wurden. 
TABELLE 10: Akademischer Grad und Diffusionschance

\begin{tabular}{lrrrrc}
\hline & \multicolumn{2}{c}{ Einger. } & \multicolumn{2}{c}{ Veröffentl. } & Diff.-Chance \\
& $\mathrm{N}$ & \multicolumn{1}{c}{$\%$} & \multicolumn{1}{c}{$\mathrm{N}$} & $\%$ & \multicolumn{1}{c}{$\%$} \\
\hline Ohne Grad/K.A. & 81 & 12,6 & 19 & 7,5 & 23,5 \\
Magister & 15 & 2,3 & 4 & 1,6 & 26,7 \\
Diplom & 63 & 9,8 & 25 & 9,8 & 39,7 \\
Doktor & 314 & 48,8 & 117 & 46,1 & 37,3 \\
Priv.-Doz. & 20 & 3,1 & 10 & 3,9 & 50,0 \\
Professor & 149 & 23,1 & 77 & 30,3 & 51,7 \\
Andere & 2 & 0,3 & 2 & 0,8 & 100,0 \\
\hline
\end{tabular}

$644100,0254100,0$

Durchschnittl. Diff-Chance: 39,44

hohen akademischen Grad im Durchschnitt eine höhere Belohnung erfahren als Autoren, die bis zur Veröffentlichung geringere akademische Weihen erhalten haben. Tabelle 10 scheint diese These nahezu bruchlos zu bestätigen. Die Diffusionschance steigt von 23,5\% für die Autoren ohne akademischen Grad bis zu 51,7\% für Professoren an. Lediglich zwischen diplomierten und promovierten Autoren gibt es eine Diskontinuität; der Abstand beider Annahmeraten ist jedoch gering.

Widerspricht dieses Ergebnis nun der universalistischen Norm, daß der Rang eines Autors ohne Einfluß auf die Annahme sein sollte? Die Frage läßt sich anhand der vorliegenden Daten nicht beantworten, da nicht entschieden werden kann, ob die überdurchschnittlichen Annahmeraten innerhalb der höheren Ränge auf die besondere Qualität dieser Beiträge zurückgeht oder ob hier tatsächlich partikularistische Momente eine Rolle spielen. Vermutlich werden beide Faktoren einen Einfluß ausüben. Dem möglichen Bonus höherer Chargen stehen aber immer noch hoffnungsvolle Diffusionschancen für Autoren geringeren Ranges gegenüber, die noch etwa doppelt so hoch sind wie die durchschnittlichen Annahmeraten in den großen amerikanischen Journals. Damit besteht trotz allem noch ein deutliches Innovationspotential durch jüngere Autoren bzw. durch den akademischen Mittelbau, der uberhaupt das dominante Rekrutierungsfeld der Zeitschriften bildet.

\subsection{Alice im Wunderland: Zur Diskriminierung weiblicher Autoren}

Der möglichen Diskriminierung der Frau in der Wissenschaft und speziell in der Soziologie ist in zahlreichen Studien nachgegangen worden. Der
Beitrag von Patterson (1971) ist nur ein Beispiel von vielen. Die Autorin kommt zu folgendem Ergebnis: "Women are excluded from high-ranking departments and they are excluded from highranking positions in almost all departments. The data convincingly demonstrate that these exclusions are not justified by personal qualifications" (Patterson 1971: 232). Die Autorin stützt sich bei ihrer Analyse auf das "Directory" der American Sociological Association und auf den "Guide to Graduate Departments of Sociology". Wie kann man aber Diskriminierung überzeugend belegen, wenn man den Bestand auszählt? Eine Disproportionalität bedeutet nicht notwendig eine Diskriminierung bei der Besetzung von Positionen. Innerhalb des in Frage kommenden Personals sind möglicherweise Frauen schwächer vertreten. Das verweist auf mögliche Diskriminierungen auf früheren Ausbildungsstufen.

Deshalb indiziert ein Anteil von 4,7\% der veröffentlichten Aufsätze in der Zeitschrift für Soziologie, die eine Frau als ersten Autor aufweisen, noch keine Diskriminierung (Tab. 11).

TABELLE 11: Geschlecht und Diffusionschance

\begin{tabular}{lrrrrr}
\hline & \multicolumn{4}{c}{ Eingereicht } & \multicolumn{3}{c}{ Veröffentl. } & Diff.-Chance \\
& $\mathrm{N}$ & \multicolumn{1}{c}{$\%$} & \multicolumn{1}{c}{$\mathrm{N}$} & \multicolumn{1}{c}{$\%$} & $\%$ \\
\hline Männlich & 578 & 89,8 & 242 & 95,3 & 41,9 \\
Weiblich & 59 & 9,2 & 12 & 4,7 & 20,3 \\
Unbekannt & 7 & 1,1 & 0 & 0,0 & 0,0 \\
\hline & 644 & 100,0 & 254 & 100,0 \\
Diff.-Chance & & & 39,44 \\
\hline
\end{tabular}

Dieser geringe Anteil - 12 Beiträge in $9 \mathrm{Jahr}$ gängen! - dürfte aus der geringen Vertretung weiblicher Wissenschaftler innerhalb der Soziologie resultieren. Tatsächlich betrug der Anteil der Frauen unter den Professoren 1974 (H2 bis H4) ganze 9,5\% (29 von 306), und 1979 war sogar ein geringerer Anteil zu verzeichnen, nämlich $8,1 \%$ (41 von 505; zu den Daten vgl. Sahner 1981: 58ff). Allerdings haben unter den eingereichten Manuskripten 9,2\% einen weiblichen ersten Autor (Tab. 11). Hier deutet sich eine geringere Annahmechance der Beiträge weiblicher Autoren und damit eine Diskriminierung an. Während die Autoren eine Diffusionschance von 41,9\% haben, liegen die Autorinnen mit 20,3\% weit unter dem Durchschnitt. 
Wir haben jedoch gesehen, daß die Publikationschance mit dem Status variiert. Daher könnte die geringere Publikationschance der Frauen durch einen durchschnittlich geringeren akademischen Status zu erklären sein. Tab. 12 zeigt die Publikationschance für die verschiedenen akademischen Grade getrennt nach Frauen und Männern. Der Vergleich der qualifikationsspezifischen Diffusionschancen für die Geschlechter ist aufgrund der geringen Fallzahl bei den Frauen nicht möglich bzw. sinnvoll. Wendet man jedoch die für die Männer beobachteten qualifikationsspezifischen Publikationschancen für die Frauen an, dann müßten von den 59 Manuskripten der Frauen rund 23 angenommen worden sein, was einer Diffusionschance von 39\% entspräche. Die Differenz zu den 41,9\% der Männer ginge auf das Konto der im Durchschnitt geringeren Qualifikation der Frauen. Der im Durchschnitt geringere Status der weiblichen Autoren kann also nur $\mathrm{zu}$ einem verschwindend geringen Anteil für die niedrige Diffusionschance von $20,3 \%$ verantwortlich gemacht werden.

Selbstverständlich kann der relative Mißerfolg weiblicher Autoren auch noch auf andere Fak. toren zurückgeführt werden, so z.B. auf die geringe Qualität der eingereichten Arbeiten oder auf die falschen Forschungsfelder. Es bleibt aber auch der Verdacht, daß hier partikularistische Momente bei der Bewertung eine Rolle gespielt haben: nicht nur das Ergebnis wird bewertet, sondern auch die Person.

\section{Resümee}

Reproduzieren die Autoren und die Herausgeber in der Zeitschrift den Spiegel des eigenen
Faches? Zumindest nach der Analyse dieser einnen Zeitschrift muß man es bezweifeln. Die Herausgeber haben einen weiten Spielraum zur Selektion und sie nutzen ihn auch. Gegen diesen Sachverhalt selbst ist auch nichts einzuwenden, denn ansonsten könnte man die zu veröffentlichenden Aufsätze auch nach dem Zufallsprinzip auswählen. Die Frage ist nur, ob die Selektionsprinzipien mit den Normen der Profession vereinbar sind.

Die Einzelergebnisse sollen hier nicht noch einmal aufgeführt werden. Nur drei wichtige Problemkreise seien hervorgehoben. Die Analyse konnte einmal zumindest die Herausgeber der Zeitschrift für Soziologie von den allgemein erhobenen Vorwürfen befreien, sie trügen durch ihre Herausgeberpolitik zur Artefaktbildung bei, indem signifikante Ergebnisse und Bestätigungen eine größere Annahmechance hätten als die gegenteiligen Ergebnisse. Die Einzelanalyse der Aufsätze festigt aber den Verdacht, daß zumindest unter der Autorenschaft signifikante Ergebnisse und Bestätigungen auf besonderes Wohlwollen stoßen. Vielleicht wirken die Ergebnisse dieser Studie positiv auf die Autoren zurück.

Es lassen sich sachliche Präferenzen der Herausgeber feststellen, die aber verbreitete Anerkennung finden dürften, so wenn sie makrosoziologische Fragestellungen fördern und die Dominanz des Theoretisierens, sei cs in der Form der Meta-Theorie oder der Dokumentation literarischer Belesenheit, der endlosen Interpretation der Gründerväter etc. abzubauen suchen. Die gewünschte Förderung der Substanzforschung gelingt ihnen jedoch nicht. Deutliche Präferenzen der Herausgeber zeichnen sich hinsicht-

TABELLE 12: Akademischer Grad und geschlechtsspezifische Diffusions-Chance

\begin{tabular}{|c|c|c|c|c|c|c|c|c|c|c|c|c|c|c|c|}
\hline & \multicolumn{2}{|c|}{ Einger. } & \multicolumn{2}{|c|}{$\begin{array}{c}\text { Männlich } \\
\text { Abgel. }\end{array}$} & \multicolumn{2}{|c|}{ Veröff. } & \multirow{2}{*}{$\begin{array}{c}\text { Diff.- } \\
\text { Chance } \\
\%\end{array}$} & \multicolumn{2}{|c|}{ Einger. } & \multicolumn{2}{|c|}{$\begin{array}{l}\text { eiblich } \\
\text { Abgel. }\end{array}$} & \multicolumn{2}{|c|}{ Veröff. } & \multirow{2}{*}{$\begin{array}{c}\text { Diff.- } \\
\text { Chance } \\
\qquad \%\end{array}$} & \multirow{2}{*}{$\begin{array}{l}\text { Veröff. nach } \\
\text { Diff.-Chance } \\
\text { der Männer } \\
\text { N }\end{array}$} \\
\hline & $\mathbf{N}$ & $\%$ & $\mathbf{N}$ & $\%$ & $\mathbf{N}$ & $\%$ & & $\mathbf{N}$ & $\%$ & $\mathbf{N}$ & $\%$ & $\mathbf{N}$ & $\%$ & & \\
\hline Ohne Grad/K.A. & 63 & 10,9 & 45 & 13,4 & 18 & 7,4 & 28,6 & 15 & 25,4 & 14 & 29,8 & 1 & 8,3 & 6,7 & 4,29 \\
\hline Magister & 12 & 2,1 & 8 & 2,4 & 4 & 1,7 & 33,3 & 3 & 5,1 & 3 & 6,4 & 0 & 0,0 & 0,0 & 1,00 \\
\hline Diplom & 59 & 10,2 & 34 & 10,1 & 25 & 10,3 & 42,4 & 4 & 6,8 & 4 & 8,5 & 0 & 0,0 & 0,0 & 1,70 \\
\hline Doktor & 283 & 49,0 & 172 & 51,2 & 111 & 45,9 & 39,2 & 28 & 47,5 & 22 & 46,8 & 6 & 50,0 & 21,4 & 10,98 \\
\hline Priv.Doz. & 18 & 3,1 & 8 & 2,4 & 10 & 4,1 & 55,6 & 2 & 3,4 & 2 & 4,3 & 0 & 0,0 & 0,0 & 1,11 \\
\hline Professor & 141 & 24,4 & 69 & 20,5 & 72 & 29,8 & 51,1 & 7 & 11,9 & 2 & 4,3 & 5 & 41,7 & 71,4 & 3,58 \\
\hline \multirow[t]{2}{*}{ Andere } & 2 & 0,3 & 0 & 0,0 & 2 & 0,8 & 100,0 & $\mathbf{0}$ & 0,0 & 0 & 0,0 & 0 & 0,0 & - & - \\
\hline & 578 & 100,0 & 336 & 100,0 & 242 & 100,0 & & 59 & & 47 & 100,0 & 12 & 100,0 & & 22,66 \\
\hline
\end{tabular}


lich der modelltheoretischen Fundierung ab, und hier erfährt besonders das naturwissenschaftliche Verständnis Förderung, während eine verstehende Soziologie und erst recht eine marxistisch orientierte Soziologie in unterdurchschnittlichem Maße diffundiert.

Schließlich kann man partikularistische Tendenzen hinsichtlich der Merkmale Hochschulort,
Status und Geschlecht vermuten. Dies zeigt z.B. auch, mit welcher Vorsicht man der Behauptung begegnen muß, daß die drei großen Periodika nicht auf eine personelle Begünstigung des eigenen Instituts ausgerichtet sind. Diese Vorsicht ist auch deshalb angebracht, weil die Zeitschrift für Soziologie durch ihre Organisation am wenigsten dieser Gefahr unterliegen dürfte.

\section{Anhang}

Randverteilung der Forschungsfelder für den detaillierten Code (1, inhaltlicher Schwerpunkt)

\begin{tabular}{|c|c|c|c|c|c|}
\hline Inhaltlicher Schwerpunkt & Code & $\begin{array}{l}\text { Eingereichte } \\
\mathrm{N}\end{array}$ & $\begin{array}{l}\text { Arbeiten } \\
\%\end{array}$ & $\begin{array}{l}\text { Veröffentlichte } \\
\mathrm{N}\end{array}$ & $\begin{array}{l}\text { Arbeiten } \\
\%\end{array}$ \\
\hline \multicolumn{6}{|l|}{ Methoden } \\
\hline Datenerhebung & 1 & 14 & 2.2 & 5 & 2.2 \\
\hline Datenanalyse & 2 & 28 & 4.3 & 16 & 6.3 \\
\hline Auswahlverfahren & 3 & 1 & 0.2 & 0 & 0.0 \\
\hline Meß- u. Skalierungsverfahren & 4 & 18 & 2.8 & 11 & 4.3 \\
\hline Forschungsprozeß & 5 & 4 & 0.6 & 2 & 0.8 \\
\hline EDV & 6 & 1 & 0.2 & 1 & 0.4 \\
\hline Sonstige & 7 & 13 & 2.0 & 6 & 2.4 \\
\hline Wissenschaftstheorie & 8 & 50 & 7.8 & 18 & 7.1 \\
\hline \multicolumn{6}{|l|}{ Theorie } \\
\hline Theorie allgemein & 9 & 15 & 2.3 & 4 & 1.6 \\
\hline Strukturfunktionalismus & 10 & 8 & 1.2 & 5 & 2.0 \\
\hline Systemtheorie & 11 & 16 & 2.5 & 8 & 3.1 \\
\hline Konflikt theorie & 12 & 2 & 0.3 & 0 & 0.0 \\
\hline Handlungstheorie & 13 & 6 & 0.9 & 4 & 1.6 \\
\hline Symbol. Interaktionismus & 14 & 5 & 0.8 & 2 & 0.8 \\
\hline Ethnomethodologie & 15 & 1 & 0.2 & 1 & 0.4 \\
\hline Verhaltenstheorie & 16 & 8 & 1.2 & 6 & 2.4 \\
\hline Marxist. Theorie & 17 & 4 & 0.6 & 2 & 0.8 \\
\hline Mathem. Soziologie & 18 & 9 & 1.4 & 8 & 3.1 \\
\hline Sonstige Ansätze & 19 & 9 & 1.4 & 0 & 0.0 \\
\hline Geschichte der Soz. & 20 & 0 & 0.0 & 0 & 0.0 \\
\hline Einzelne Klassiker & 21 & 30 & 4.7 & 15 & 5.9 \\
\hline Sozialphilosophie & 22 & 4 & 0.6 & 0 & 0.0 \\
\hline \multicolumn{6}{|l|}{ Makrosoziol/Gegenwartsges. } \\
\hline Makrosoziologie allg. & 23 & 1 & 0.2 & 1 & 0.4 \\
\hline Mod./Ind. Gesellschaften & 24 & 10 & 1.6 & 8 & 3.1 \\
\hline Bürgerl./Kapital. Ges. & 25 & 2 & 0.3 & 0 & 0.0 \\
\hline Ges. d. BRD & 26 & 0 & 0.0 & 0 & 0.0 \\
\hline Ges. d. DDR & 27 & 1 & 0.2 & 1 & 0.4 \\
\hline andere kap. Ges. & 28 & 1 & 0.2 & 0 & 0.0 \\
\hline andere sozial. Ges. & 29 & 1 & 0.2 & 1 & 0.4 \\
\hline sonst. Makrosoziologie & 30 & 1 & 0.2 & 1 & 0.4 \\
\hline \multicolumn{6}{|l|}{ Schichtung } \\
\hline Schichtung & 31 & 9 & 1.4 & 4 & 1.6 \\
\hline Klasse & 32 & 1 & 0.2 & 0 & 0.0 \\
\hline Mobilität & 33 & 4 & 0.6 & 4 & 1.6 \\
\hline Arbeiterklasse & 34 & 1 & 0.2 & 1 & 0.4 \\
\hline
\end{tabular}


Inhaltlicher Schwerpunkt

Code Eingereichte Arbeiten

Veröffentlichte Arbeiten $\mathrm{N}$

$\%$

$\%$

Sozialer Wandel

Sozialer Wandel

Industrialisierung

Soziale Bewegungen

35

36

37

Entwicklungsländer

Soz. Instit. u. Organis.

Institutionen

Organisationen

Bürokratie

Wirtschaftssoziologie

allgemein

Industrie u. Betrieb

Arbeit und Beruf

Sonstiges

Politische Ökonomie

Politische Soziologie

allgeme in

Macht, Herrschaft

Staat

Parteien, Verbände

Wahlen

Demokratie

Totalit., Dikt., Faschismus

Öffentl. Verwaltung

Planung, Verwaltung

Internat. Beziehungen

Revolution

Sonstiges

Rechtssoziologie

Erziehungssoziologie

Wissenschaftssoziologie

allgemein

Soziologie d. Soziol.

Medizinsoziologie

Regionalsoziologie

Agrarsoziologie

Stadt-, Gemeinde-Soz.

Regionalsoziologie

Stadt-, Raumplanung etc.

Sportsoziologie

Religionssoziologie

Kultursoziologie

allgemein

Wissenssoziologie

Ideologie

Kunst, Literatur, Musik

Sprache
39

40

41

42

43

44

45

46

47

48

49

50

51

52

53

54

55

56

57

58

59

60

61

62

63

64

65

66

67

68

69

70

71

72

73

74
0.6

0.5

0.5

1.1

0.0

1.1

0.3

0.0

0.9

3.7

1.9

0.3

0.0

0.3

0.5

1.4

0.6

0.3

0.3

0.5

0.5

0.2

0.0

0.6

1.9

4.3

4.3

1.9

2.6

0.0

0.3

0.6

0.8

1.4

0.5

0.5

0.0

0.2

1.1

0.5
0.4

1.2

0.4

0.4

0.0

1.2

0.4

0.0

0.4

1.6

1.2

0.4

0.0

0.0

0.4

0.4

0.4

0.0

0.0

0.8

0.0

0.4

0.0

0.0

1.6

3.5

5.5

1.2

2.4

0.0

0.0

1.2

0.8

1.6

0.0

0.4

0.0

0.0

0.4

0.0 


\begin{tabular}{|c|c|c|c|c|c|}
\hline Inhaltlicher Schwerpunkt & Code & \multicolumn{2}{|c|}{$\begin{array}{l}\text { Eingereichte Arbeiten } \\
\mathrm{N}\end{array}$} & \multicolumn{2}{|c|}{$\begin{array}{l}\text { Veröffentlichte Arbeiten } \\
\mathrm{N}\end{array}$} \\
\hline \multicolumn{6}{|l|}{ Familie etc. } \\
\hline Familiensoziologie & 75 & 7 & 1.1 & 5 & 2.0 \\
\hline Jugendsoziologie & 76 & 7 & 1.1 & 4 & 1.6 \\
\hline Familie und Jugend & 77 & 2 & 0.3 & 2 & 0.8 \\
\hline Alter, Tod & 78 & 0 & 0.0 & 0 & 0.0 \\
\hline Frauenfragen & 79 & 8 & 1.2 & 2 & 0.8 \\
\hline \multicolumn{6}{|l|}{ Sozialpsychologie etc. } \\
\hline Massenverhalten & 81 & 0 & 0.0 & 0 & 0.0 \\
\hline Massenkommunikation & 82 & 6 & 0.9 & 2 & 0.8 \\
\hline Gruppe & 83 & 8 & 1.2 & 2 & 0.8 \\
\hline Einstellung, Vorurteil & 84 & 12 & 1.9 & 4 & 1.6 \\
\hline Minoritäten & 85 & 0 & 0.0 & 0 & 0.0 \\
\hline Sozialisation & 86 & 20 & 3.1 & 5 & 2.0 \\
\hline Psych., Psychoanalyse & 87 & 4 & 0.6 & 0 & 0.0 \\
\hline Sonstiges & 88 & 9 & 1.4 & 4 & 1.6 \\
\hline \multicolumn{6}{|l|}{ Soziale Probleme } \\
\hline allgemein/Sonstiges & 89 & 2 & 0.3 & 0 & 0.0 \\
\hline abweichendes Verhalten & 90 & 14 & 2.2 & 4 & 1.6 \\
\hline Gastarbeiter & 91 & 9 & 1.4 & 3 & 1.2 \\
\hline Freizeit & 92 & 1 & 0.2 & 0 & 0.0 \\
\hline Sozialpolitik, Planung & 93 & 7 & 1.1 & 5 & 2.0 \\
\hline Sozialarbeit & 94 & 3 & 0.5 & 2 & 0.8 \\
\hline Sozialanthropologie & 95 & 4 & 0.6 & 2 & 0.8 \\
\hline Sozialgeschichte & 96 & 2 & 0.3 & 1 & 0.4 \\
\hline Christliche Soziallehre & 97 & 0 & 0.0 & 0 & 0.0 \\
\hline \multirow[t]{2}{*}{ Sonstige/nicht klassifiziert } & 98 & 3 & 0.5 & 0 & 0.0 \\
\hline & & 644 & 100.0 & 254 & 100.0 \\
\hline
\end{tabular}

\section{Literatur:}

Baier, H./Kaufmann, F.-X./Klima, R./Oevermann, U./ Schoene, W., 1972: Vorwort der Herausgeber. Zeitschrift für Soziologie 1: 2-4.

Baier, H./Kaufmann, F.-X./Klima, R./Oevermann, U./ Schoene, W., 1974: Zur Eröffnung des dritten Jahrgangs - Vorwort der Herausgeber. Zeitschrift für Soziologie 3: 1-4.

Bakan, David, 1966: The Test of Significance in Psychological Research. Psychological Bulletin 66, No. 6: 423-437.

Becker, H.S., 1970: Sociological Work. Method and Substance. Chicago.

Beyer, Janice M., 1978: Editorial Policies and Practices Among Leading Journals in Four Scientific Fields. The Sociological Quarterly 19: 68-88.

Bloxham, M., 1976: A Note on Publication and the Value of Significance Tests. Theory and Decision $7: 135-139$.

Bolte, Karl Martin, 1978: Vortrag zur Eröffnung des 18. Deutschen Soziologentages: Zur Situation soziologischer Forschung und Lehre in der Bundes- republik Deutschland. In: Materialien aus der soziologischen Forschung. Verhandlungen des 18. Deutschen Soziologentages vom 28. September bis 1. Oktober 1976 in Bielefeld, hersg. von Karl Martin Bolte. München: Deutsche Gesellschaft für Soziologie.

Bredenkamp, Jürgen/Feger, Hubert, 1970: Kriterien für die Entscheidung über die Aufnahme empirischer Arbeiten in die Zeitschrift für Sozialpsychologie. Zeitschrift für Sozialpsychologie 1: 43-47.

Brown, Julia S./Gilmartin, Brian G., 1969: Sociology Today: Lacunae, Emphases, and Surfeits. The American Sociologist 4: 283-291.

Cole, Jonathan R./Cole, Stephen, 1973: Social Stratification in Science. Chicago: University of Chicago Press.

Crane, D., 1967: Gatekeepers of science: Some factors affecting the selection of articles for scientific publication. American Sociologist 2: 195-201.

Eisermann, Gottfried (Hrsg.), 1976: Die Krise der Soziologie. Stuttgart: Enke. 
Flora, P./Harder, Th./Klima, R./Lipp, W./Sprondel, W.M., 1981: Zur Eröffnung des zehnten Jahrgangs - Vorwort der Herausgeber. Zeitschrift für Soziologie 10: 1-6.

Friedrichs, Robert W., 1970: A Sociology of Sociology. New York: Free Press.

Galtung, Johan, 1967: Theory and Methods of Social Research. Oslo: Universitetsforlaget.

Gaston, Jerry (Hrsg.), 1978: Sociology of Science. San Francisco/Washington/Londen: Jossey-Bass

Gaston, Jerry, 1979: The Big Three and the Status of Sociology. Contemporary Sociology 8: 789-793.

Gouldner, Alvin W., 1974: Die westliche Soziologie in der Krise. Reinbek bei Hamburg: Rowohlt.

Greenwald, Anthony G., 1975: Consequences of Prejudice Against the Null Hypothesis. Psychological Bulletin 82, No. 1: 1-20.

Hartmann, Heinz, 1979: Zum Eintritt in den 30. Jahrgang. Bilanz und Ausblick für die Zeitschrift ,Soziale Welt". Soziale Welt 30: 1-8.

Hauck, Gerhard, 1972: Unhistorische „Prozesse sozialer Mobilisierung". Bemerkungen zu P. Flora. Zeitschrift für Soziologie 1: 377-379.

Kaufmann, F.-X./Klima, R./Oevermann, U./Schluchter, W./Schoene, W., 1975: Zur Eröffnung des vierten Jahrgangs - Vorwort der Herausgeber. Zeitschrift für Soziologie 4, 1-5.

Klima, Rolf, 1979: Die Entwicklung der soziologischen Lehre an den westdeutschen Universitäten 19501975. Eine Analyse der Vorlesungsverzeichnisse. S. 221-256 in: G. Lüschen (Hrsg.), Deutsche Soziologie seit 1945. Opladen.

König, René, 1969: Einige Bemerkungen über die Bedeutung der empirischen Forschung in der Soziologie. S. 1278-1291 in: R. König (Hrsg.), Handbuch der empirischen Sozialforschung, Zweiter Band. Stuttgart: Enke.

König, René, 1979: Einige Bemerkungen über die Bedeutung der empirischen Forschung für die Soziologie. S. 345-375 in R. König (Hrsg.), Handbuch der empirischen Sozialforschung, Bd. 14. Stuttgart: Enke.

Krysmanski, H.J./Marwedel, P. (Hrsg.), 1975: Die Krise in der Soziologie. Ein kritischer Reader zum 17. Deutschen Soziologentag. Köln.

Lepsius, M. Rainer 1979: Die Entwicklung der Soziologie nach dem zweiten Weltkrieg. S. 25-70 in: Günter Lüschen (Hrsg.), Deutsche Soziologie seit 1945, Opladen.

Lindsey, D., 1978: The Scientific Publication System in Social Science: A Study of the Operation of Leading Professional Journals in Psychology, Sociology, and Social Work. San Francisco: Jossey-Bass.

Luckmann, Thomas, 1974: Das kosmologische Fiasko. Soziologie 2: $15-32$

Lüschen, Günther, 1979: Die Entwicklung der deutschen Soziologie in ihrem Fachschriftentum. Perioden, Sachgebiete und Methoden seit 1945. S. 169-192 in: G. Lüschen (Hrsg.), Deutsche Soziologie seit 1945. Opladen.

Matthes, Joachim 1978: Die Diskussion um den Theorienvergleich in den Sozialwissenschaften seit dem Kasseler Soziologentag 1974. S. 7-20 in: Karl-Otto Hondrich/Joachim Matthes (Hrsg.), Theorienvergleich in den Sozialwissenschaften. Darmstadt und Neuwied: Luchterhand.

Melton, A.W., 1962: Editorial Journal of Experimental Psychology 64: 553-557.

Merton, Robert K., 1972: Wissenschaft und demokratische Sozialstruktur. S. 45-59 in: Wissenschaftssoziologie 1. Wissenschaftliche Entwicklung als sozialer Prozeß, hersg. von Peter Weingart. Frankfurt: Fischer Athenäum.

Merton, Robert K., 1973: The Sociology of Science. Theoretical and Empirical Investigations. Edited and with an Introduction by Norman W. Storer. Chicago und London: The University of Chicago Press.

Neidhardt, Friedhelm, 1976: Identitäts- und Vermittlungsprobleme der Soziologie. In: Zwischenbilanz der Soziologie, Verhandlungen des 17 . Soziologentages, hrsg. von M. Rainer Lepsius. Stuttgart.

Neues Testament, übersetzt und erklärt von Otto Karrer. München 1954: Ars Sacra.

Patterson, Michelle, 1971: Alice in Wonderland: A Study of Women Faculty in Graduate Departments of Sociology. The American Sociologist 6: 226-234.

Pfeffer, Jeffrey/Leong, Antony/Strehl, Katherine, 1977: Paradigm Development and Particularism: Journal Publication in Three Scientific Disciplines. Social Forces 55: 938-951.

Sahner, Heinz, 1979: Veröffentlichte empirische Sozialforschung: Eine Kumulation von Artefakten? Eine Analyse von Periodika. Zeitschrift für Soziologie 8: 267-278.

Sahner, Heinz, 1981: Theorie und Forschung. Zur paradigmatischen Struktur der westdeutschen Soziologie und zu ihrem Einfluß auf die Forschung. Kiel (Habilitationsschrift).

Schelsky, Helmut, 1950: Lage und Aufgabe der angewandten Soziologie in Deutschland. Soziale Welt 1: 3-14.

Simon, Rita J., 1980: 1980 Editors' Reports. American Sociological Association Footnotes 8, No. 3: 10.

Sterling, Theodore D., 1959: Publication Decisions and their Possible Effects on Inferences Drawn from Tests of Significance - or Vice Versa. American Statistical Association Journal 54: 30-34.

Whitley, Richard D., 1970: The Operation of Science Journals: Two Case Studies in British Social Science. The Sociological Review 18: 241-258.

Wiley, Norbert, 1979: Recent Journal Sociology: The Substitution of Method for Theory. Contemporary Sociology 8, 793-799.

Wilson, Franklin D./Smoke, Gale L./Martin, J. David, 1973: The Replication Problem in Sociology: A Report and a Suggestion. Sociological Inquiry 43: $141-149$.

Yoels, William C., 1974: The Structure of Scientific Fields and the Allocation of Editorship on Scientific Journals: Some Observations on the Politics of Knowledge. The Sociological Quarterly 15: 264276.

Zelditch, Morris, Jr., 1979: Why Was the ASR So Atheoretical? Contemporary Sociology 8: 808-813.

Zuckerman, Harriet, 1978: Theory Choice and Problem Choice in Science. In: Jerry Gaston (Hrsg.), Sociology of Science. San Francisco/Washington/London: Jossey-Bass. 